\title{
Enhanced Efficacy without Further Cleft Closure: Reevaluating Twist as a Source of Agonist Efficacy in AMPA Receptors
}

\author{
Amanda Birdsey-Benson, ${ }^{1 *}$ Avinash Gill, ${ }^{1 \star}$ Leslie P. Henderson, ${ }^{2}$ and Dean R. Madden ${ }^{1}$ \\ Departments of ${ }^{1}$ Biochemistry and ${ }^{2}$ Physiology, Dartmouth Medical School, Hanover, New Hampshire 03755
}

\begin{abstract}
AMPA receptors (AMPARs) are tetrameric ligand-gated ion channels that couple the energy of glutamate binding to the opening of a transmembrane channel. Crystallographic and electrophysiological analysis of AMPARs has suggested a coupling between (1) cleft closure in the bilobate ligand-binding domain (LBD), (2) the resulting separation of transmembrane helix attachment points across subunit dimers, and (3) agonist efficacy. In general, more efficacious agonists induce greater degrees of cleft closure and transmembrane separation than partial agonists. Several apparent violations of the cleft-closure/efficacy paradigm have emerged, although in all cases, intradimer separation remains as the driving force for channel opening. Here, we examine the structural basis of partial agonism in GluA4 AMPARs. We find that the L651V substitution enhances the relative efficacy of kainate without increasing either LBD cleft closure or transmembrane separation. Instead, the conformational change relative to the wild-type:kainate complex involves a twisting motion with the efficacy contribution opposite from that expected based on previous analyses. As a result, channel opening may involve transmembrane rearrangements with a significant rotational component. Furthermore, a two-dimensional analysis of agonist-induced GluA2 LBD motions suggests that efficacy is not a linearly varying function of lobe 2 displacement vectors, but is rather determined by specific conformational requirements of the transmembrane domains.
\end{abstract}

\section{Introduction}

Glutamate is the most common neurotransmitter in the brain, mediating signals at $>80 \%$ of the synapses in the cortex (Braitenberg and Schüz, 1998). Among the postsynaptic ionotropic glutamate receptors (iGluRs) that respond to glutamatergic signals, the subfamily selective for AMPA ( $\alpha$-amino-3-hydroxy-5methyl-4-isoxazole propionate) plays key roles both in excitatory neuronal communication and in learning and memory (Dingledine et al., 1999; Derkach et al., 2007). They are also implicated in a wide variety of pathological conditions and represent potentially important therapeutic targets.

The initial step of iGluR signal transduction involves the interaction of glutamate with the extracellular ligand-binding domain (LBD) found in each of the four subunits [GluA1-4, also known as GluRA-D or GluR1-4 (Collingridge et al., 2009)] that can assemble to form functional tetrameric AMPA receptors (AMPARs). Biophysical studies of the LBD have shown that agonist binding stabilizes a Venus-flytrap closure of two lobes that

Received Sept. 14, 2009; revised Dec. 4, 2009; accepted Dec. 9, 2009.

This work was supported in part by grants from the Hitchcock Foundation (to D.R.M.) and the National Institutes of Health (R01-GM075310 to D.R.M., R01-DA014137 to L.P.H., and T32-DK007301 to A.B.-B.). A.G. was supported by a Copenhaver and Thomas Fellowship and by the Rosaline Borison Memorial Fund. We thank Dr. P. Seeburg (Max Planck Institute, Heidelberg) and Dr. D. Bowie (McGill University, Montréal, QC, Canada) for reagents, and Drs. R. Maue, B. L. Jones, and J. Kull (Dartmouth College, Hanover, NH) for advice and discussions.

${ }^{*}$ A.B.-B. and A.G. contributed equally to this work.

Correspondence should be addressed to Dean R. Madden, Department of Biochemistry, Dartmouth Medical School, 7200 Vail Building, Hanover, NH 03755. E-mail: drm0001@dartmouth.edu.

D01:10.1523/JNEUROSCI.4558-09.2010

Copyright $\odot 2010$ the authors $\quad 0270-6474 / 10 / 301463-08 \$ 15.00 / 0$ surround the ligand-binding site (Madden, 2002). Furthermore, the magnitude of this generative conformational change is variable and correlates with the efficacy of the bound agonist (Armstrong and Gouaux, 2000). It has also been observed that the LBDs dimerize back to back across an interface formed by the membrane-distal lobes of the domain. As a result, cleft closure in both monomers pulls apart the membrane-proximal lobes. This motion increases the distance between the linker peptides that replace the first two transmembrane (TM) domains in the recombinant construct. Linker separation has therefore been considered a surrogate for the conformational changes in the pore associated with channel activation.

Recent experiments indicate that the cleft-closure hypothesis is incomplete. First, a fully closed LBD can still be associated with partial agonism (Inanobe et al., 2005; Zhang et al., 2008; Frydenvang et al., 2009). Second, LBD cleft closure can be accompanied by an orthogonal "twisting" motion (Holm et al., 2005). In particular, a retrospective analysis of published LBD structures suggested that efficacy was reduced by a clockwise twist of the bottom lobe relative to the upper lobe, viewed from the perspective of the transmembrane domains (Bjerrum and Biggin, 2008). The twist component was proposed to act by modifying the extent of linker separation associated with cleft closure.

A key regulator of the extent of cleft closure in the GluA2 subunit is Leu650. Mutation to the smaller Thr side chain (GluA2-L650T) permitted increased cleft closure and relative efficacy in the presence of kainate (Armstrong et al., 2003). However, several unexpected effects were also observed, potentially associated with the polar Thr hydroxyl moiety. In addition, the 
wild-type (WT) GluA4 LBD closes almost as tightly when bound to kainate as does the GluA2-L650T domain, because of peripheral binding-site sequence differences (Gill et al., 2008). Here, we analyze the effects of the primarily steric mutation L651V in the GluA4 AMPAR subunit. The GluA4-L651V mutation increases the relative efficacy of kainate without increasing either the degree of cleft closure in the LBD or the linker separation in the dimer, leading to a reassessment of linearly variable models of iGluR activation.

\section{Materials and Methods}

Expression constructs. Full-length constructs for electrophysiological analysis were derived from the GluA4 $4_{\text {flip }}$ construct (provided by Dr. P. Seeburg, Max Planck Institute, Heidelberg, Germany), as described previously (Gill et al., 2008). The L651V mutation was introduced into the WT and L484Y backgrounds using the QuikChange kit (Stratagene). Both the coding and noncoding strands surrounding the mutation site were sequenced to verify mutations.

The LBD construct used in crystallization studies contains a thrombin-cleavable $\mathrm{N}$-terminal polyhistidine tag followed by residues 393-507 and 633-775 of the mature sequence of GluA4 $4_{\text {flip }}$ joined by a Gly-Thr linker (Gill and Madden, 2008). The L651V mutation was introduced into the WT LBD construct using the QuikChange kit and verified by DNA sequencing.

Electrophysiology. Electrophysiological measurements were performed essentially as described previously (Gill et al., 2008), using tsA201 cells (a gift from Dr. Derek Bowie, McGill University, Montreal, QC, Canada) transiently transfected with $2 \mu \mathrm{g}$ of AMPA receptor cDNA construct and $0.8 \mu \mathrm{g}$ of pGreenLantern plasmid (Invitrogen). Whole-cell and outside-out patch current responses were recorded under voltageclamp conditions $(-60 \mathrm{mV}) \sim 24$ and $48 \mathrm{~h}$ after transfection, respectively. L-Glutamate and kainate were applied using ultrafast perfusion conditions. Values are reported as the mean \pm SE. Unpaired Student's $t$ tests were performed. Unless otherwise indicated, all recordings were performed with constructs containing the L484Y mutation to inhibit desensitization.

Protein expression, purification, and crystallization. Protein expression and purification was performed as described earlier (Gill and Madden, 2008). Purified L651V protein was crystallized by vapor diffusion against 24\% (w/v) PEG 4000, $0.1 \mathrm{~m}$ sodium acetate, $\mathrm{pH}$ 4.6, $0.3 \mathrm{~m}$ ammonium sulfate (glutamate complex), or $18 \%$ (w/v) PEG 8000 , 0.1 M sodium acetate, $\mathrm{pH}$ 5.0, $0.2 \mathrm{M}$ ammonium sulfate (kainate complex). Both crystals were grown in hanging drops (1:1 protein to buffer ratio in a total drop volume of $2 \mu \mathrm{l}$ ) equilibrated against $500 \mu \mathrm{l}$ of crystallization buffer in the reservoir. The L651V-LBD:Glu crystal was cryoprotected by soaking in crystallization buffer supplemented with $10 \mathrm{~mm}$ L-glutamate and $16 \%(\mathrm{w} / \mathrm{v})$ glycerol as a cryoprotectant and vitrified by plunging into a liquid nitrogen bath. The L651V-LBD:kainate (KA) crystal was cryoprotected in a similar manner, except that the cryoprotection buffer was supplemented with $10 \mathrm{~mm}$ kainate. Complete $\mathrm{x}$-ray datasets were collected for a GluA4 LBD-L651V:Glu crystal and for a GluA4 LBDL651V:KA crystal at $100 \mathrm{~K}$ on a MAR345dtb image plate system, using Cu $\mathrm{K} \alpha$ radiation produced by a rotating anode (Rigaku) equipped with focusing mirrors (Genova) and a Cryostream 700 (Oxford Cryosystems).

Structure refinement. Data were processed using the XDS package (Kabsch, 1993). Since the GluA4-L651V:Glu crystal exhibited noncrystallographic symmetry, the $R_{\text {free }}$ test set was selected in thin shells using the program SFTOOLS. In the case of the GluA4-L651V:KA model, the $R_{\text {free }}$ test set was picked by random selection using the program
A
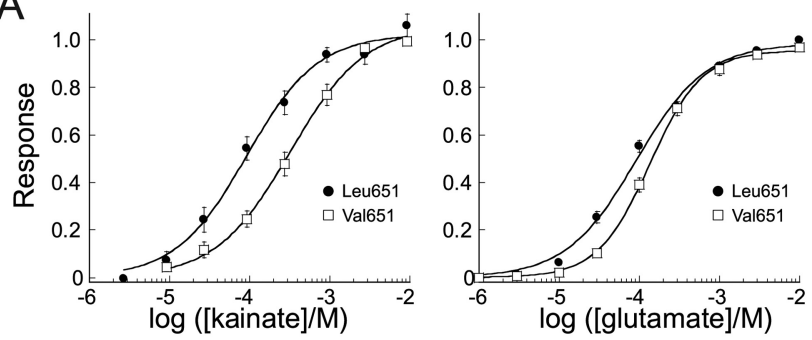

B
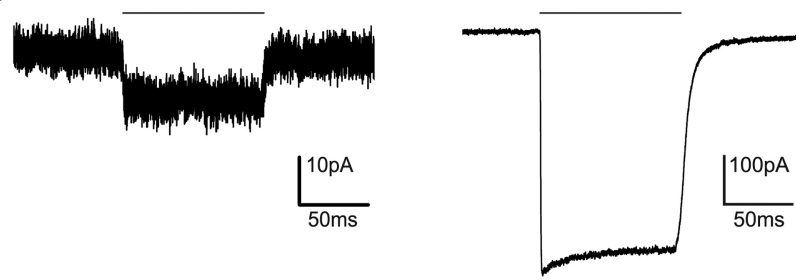

C
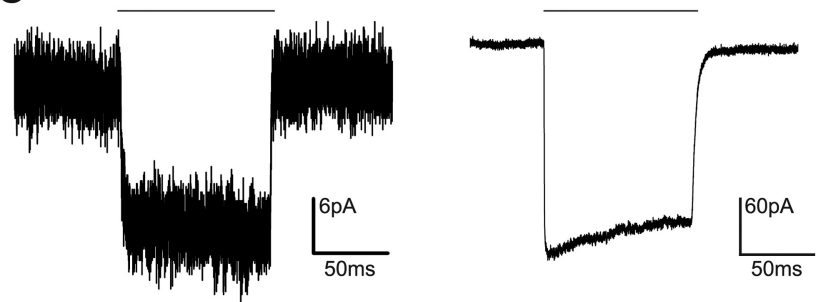

Figure 2. The L651V mutation increases the relative efficacy of kainate. $\boldsymbol{A}$, Normalized whole-cell responses to kainate (left) and glutamate (right) were measured using tsA201 cells expressing GluA4 homotetramers carrying the nondesensitizing L484Y background mutation and either Leu $(-)$ or Val $(\square)$ at position 651 , as a function of agonist concentration $(n=6$, kainate; $n=8$, glutamate). (urves were fit using the Hill equation. $B, C$, Outside-out patches obtained from cells expressing GluA4-L484Y homotetramers with either Leu $(\boldsymbol{B})$ or Val $(\boldsymbol{C})$ at position 651 were exposed to $100 \mathrm{~ms}$ pulses (horizontal bars) of $10 \mathrm{~mm} \mathrm{KA}$ (left) or $10 \mathrm{~mm}$ Glu (right), and current responses were measured under voltage-clamp conditions. Representative current recordings are shown. Note that the KA-induced currents (left) are shown at $10 \times$ scale relative to the corresponding Glu (right) responses. Averaged peak KA responses were $2.7 \pm$ $0.4 \%$ of peak glutamate responses for channels bearing the WT Leu651 side chain $(n=5)$ compared with $9.4 \pm 1.7 \%$ for channels bearing the Val651 mutation $(n=7)$. 
SFTOOLS. In each case, the same $R_{\text {free }}$ set was used throughout refinement.

The structure of the L651V-LBD:Glu cocrystal was determined first, using the program CNS (Brünger et al., 1998) to obtain initial phases by molecular replacement (MR). The search model used was the GluA4WT:Glu structure [Protein Data Bank (PDB) entry 3EPE], in which Leu651 had been truncated in silico to an alanine. Cross-rotation and translation function calculations revealed two solutions in the asymmetric unit. The MR search yielded a final correlation coefficient of 0.77 ( $15-3 \AA$ resolution) and a calculated solvent content of $42 \%$. To minimize model bias, following rigid-body refinement, simulated annealing and composite omit-map calculations were performed, together with density modification using DM (Cowtan, 1994). Initial model building, refinement, and placement of ligand molecules and waters were performed as described earlier (Gill et al., 2008). When clear density for a valine side chain was seen at the location surrounding residue 651 , the alanine was replaced with a valine.

The GluA4-L651V:KA cocrystal structure was determined by MR using the refined GluA4-L651V:Glu structure as a search model. Crossrotation and translation functions yielded a correlation coefficient of 0.61 (15 - $3 \AA$ resolution) and a calculated solvent content of $54 \%$. Calculation of density-modified composite omit maps, model building, and refinement of the GluA4-L651V:KA structure were performed using the same strategy as for the WT structure (Gill et al., 2008). $\mathrm{C}_{\alpha}$ and individual side-chain figures were prepared using CHIMERA (Pettersen et al., 2004). Ribbon diagrams were prepared using Virtual Molecular Dynamics (VMD) (Humphrey et al., 1996). Coordinates have been deposited with the PDB (entries 3KEI and 3KFM for the glutamate and kainate complexes, respectively).

Domain superpositions. Superposition of the GluA4-L651V structures with GluA4-WT and GluA2 LBD structures was performed using the program LSQKAB (Kabsch, 1976) using the fixed domain boundaries previously described for GluA2 and GluA4 (Gill et al., 2008). To visualize lobe 2 movements more comprehensively, GluA2-WT:KA (PDB entry 1FW0) and GluA4-WT:KA (PDB entry 3EN3) structures were aligned to principal axes using the Orient script in VMD (Humphrey et al., 1996). Lobe 1 of LBD structure GluA4-WT:Glu (PDB entry 3EPE) and lobe 1 of GluA4-L651V:KA were then each superimposed on GluA4-WT:KA using LSQKAB, and the positions of the lobe 2 centers of mass were calculated in projection along the first principal axis. Similar assessments were performed on the GluA2 LBD structures shown below (see Figs. 4 and 6).

\section{Results}

The L651V mutation increases the relative efficacy of kainate Analysis of the GluA4-WT LBD structure in complex with glutamate and kainate showed that Leu651 represents the primary steric constraint restricting cleft closure in the kainate-bound complex (Fig. 1). To assess the contribution of the Leu651 side chain to the relative efficacy of kainate without a confounding shift in side-chain polarity, we performed a detailed electrophysiological characterization of homomeric GluA4 channels containing either Leu (WT) or Val at position 651, using ultrafast perfusion patch-clamp techniques. To allow accurate assessment of peak current responses, desensitization was suppressed by performing measurements on subunits also carrying the nondesensitizing L484Y mutation (Gill et al., 2008). Dose-response data are shown in Figure $2 \mathrm{~A}$. Compared with receptors bearing a WT Leu side chain at position 651 (Gill et al., 2008), those bearing the L651V mutation exhibit a weaker apparent affinity for both kainate $\left(\mathrm{EC}_{50}=324 \pm 35 \mu \mathrm{M}\right.$ vs $\left.92 \pm 14 \mu \mathrm{M}, n=6\right)$ and glutamate $\left(\mathrm{EC}_{50}=136 \pm 4 \mu \mathrm{M}\right.$ vs $\left.92 \pm 10 \mu \mathrm{M}, n=8\right)$. Hill coefficients for the $\mathrm{L} 484 \mathrm{Y} / \mathrm{L} 651 \mathrm{~V}$ receptors are close to 1 (kainate $=0.9 \pm 0.1$, $n=6$; glutamate $=1.3 \pm 0.1, n=8$ ).

To determine relative efficacy for kainate, currents were recorded from outside-out patches from transfected tsA201 cells exposed successively to $10 \mathrm{~mm}$ concentrations of kainate and then glutamate. Data were obtained for nondesensitizing receptors
Table 1. Data collection and refinement statistics

\begin{tabular}{|c|c|c|}
\hline & L-Glutamate & Kainate \\
\hline \multicolumn{3}{|l|}{ Ligand } \\
\hline Space group & $P 2_{1}$ & $P 2$ \\
\hline Unit cell parameters $\left(\AA,{ }^{\circ}\right)$ & $\begin{array}{l}a=47.2, b=105.1 \\
c=66.3, \beta=97.2\end{array}$ & $\begin{array}{l}a=45.7, b=48.4 \\
c=62.8, \beta=106.6\end{array}$ \\
\hline Matthews coefficient $\left(\AA^{3} / \mathrm{Da}\right)$ & 2.86 & 2.34 \\
\hline Solvent content (\%) & 57.0 & 47.2 \\
\hline Resolution ${ }^{a}$ & $19.69-1.90(1.99-1.90)$ & $19.95-2.20(2.25-2.20)$ \\
\hline Total reflections ${ }^{a}$ & $167,093(22,257)$ & $48,592(2974)$ \\
\hline Unique reflections $^{a}$ & $45,551(6257)$ & $13,431(847)$ \\
\hline$R_{\text {sym }}{ }^{a, b}(\%)$ & $8.4(13.0)$ & $12.5(22.7)$ \\
\hline Completeness $^{a}(\%)$ & $90.2(96.1)$ & $99.3(98.6)$ \\
\hline$I / \sigma_{I}^{a}$ & $12.4(8.7)$ & $10.09(5.86)$ \\
\hline \multicolumn{3}{|l|}{ Refinement statistics } \\
\hline No. of protein atoms (non-H) & 4026 & 2013 \\
\hline No. of waters & 270 & 175 \\
\hline$R_{\text {work }}^{a}(\%)$ & $21.0(22.4)$ & $20.4(23.3)$ \\
\hline$R_{\text {free }}{ }^{a}(\%)$ & $22.5(23.4)$ & $27.9(31.2)$ \\
\hline Ramachandran plot ${ }^{\complement}(\%)$ & $93.6 / 6.4 / 0 / 0$ & $92.9 / 7.1 / 0 / 0$ \\
\hline RMSD (bonds/angles) $\left(\AA,^{\circ}\right)$ & $0.009 / 1.100$ & $0.011 / 1.302$ \\
\hline Protein $B_{\mathrm{av}}{ }^{d}\left(\AA^{2}\right)$ (chain $\left.A / B\right)$ & $10.58(10.58 / 10.58)$ & $12.83(\mathrm{NA})$ \\
\hline Ligand $B_{\mathrm{av}}{ }^{d}\left(\AA^{2}\right)($ chain $\mathrm{A} / \mathrm{B})$ & $7.87(8.58 / 7.16)$ & 9.39 (NA) \\
\hline Water $B_{\mathrm{av}}{ }^{d}\left(\AA^{2}\right)$ & 12.63 & 15.44 \\
\hline
\end{tabular}

NA, Not applicable; RMSD, root mean square deviation from targets.

${ }^{a}$ Values in parentheses indicate statistics for the highest resolution shell of data.

${ }^{b} R_{\text {sym }}=\Sigma_{\mathbf{h j}}\left|\hat{l}_{\mathbf{h}}-I_{\mathbf{h j}}\right| /\left.\Sigma_{\mathbf{h j}}\right|_{\mathbf{h j}}$.

'Core/allowed/generously allowed/disallowed.

${ }^{d}$ Residual B factors calculated by REFMAC (does not include the contribution to atomic displacements from translation, libration, and screw rotation displacement).

with either Leu or Val at position 651 (Fig. $2 B, C$ ). Kainate remains a partial agonist for channels bearing the L651V mutation but with significantly enhanced efficacy relative to glutamate. The ratio of $I_{\text {peak }}$ activated by kainate to that activated by glutamate is $9.4 \pm 1.7 \%(n=7)$ for GluA4-L484Y/L651V receptors versus $2.7 \pm 0.4 \%(n=5)$ for GluA4-L484Y receptors $(p<0.01)$. An increase in relative efficacy was also seen when currents were recorded from GluA4-L651V and GluA4-WT receptors carrying a WT desensitizing Leu484 background in the presence of 100 $\mu \mathrm{M}$ cyclothiazide (WT: $6.4 \pm 1.1 \%, n=5$; L651V: $14.3 \pm$ $2.1 \%, n=5 ; p=0.01)$.

\section{Increased efficacy is not associated with greater cleft closure or linker separation}

To determine whether the increased relative efficacy of kainate acting on GluA4-L651V receptors is associated with relaxation of the LBD cleft closure constraint, we determined the crystal structures of the GluA4-L651V LBD in complex with either glutamate or kainate at 1.9 and $2.2 \AA$, respectively (Table 1 ). As had previously been seen for both GluA2 and GluA4 WT domains (Armstrong and Gouaux, 2000; Gill et al., 2008), both agonist complexes display a bilobate structure with a clearly defined ligand-binding cleft (Fig. $3 A, C$ ). The GluA4-L651V LBD is more tightly closed when bound to glutamate than when bound to kainate.

The glutamate-bound structures of both the WT and L651V GluA4 LBD superimpose closely, with a root mean square (rms) difference of only $0.14 \AA$ between $\mathrm{C}_{\alpha}$ positions (Fig. $3 A, B$ ). A somewhat larger $0.4 \AA \mathrm{rms} \mathrm{C}_{\alpha}$ difference is observed when the kainate-bound structures are compared (Fig. $3 C, D$ ), consistent with a modest conformational shift within the domain. In principle, the change in relative efficacy described above could be caused by an increase in kainate efficacy, a decrease in glutamate 
efficacy, or a combination of these effects. However, the fact that the LBD conformational changes associated with the mutation are larger for the kainate-bound state than for the glutamate-bound state suggests that a change in kainate efficacy may be the predominant effect.

To investigate the nature of the conformational change further, we analyzed the position of lobe 2 following superposition of the core residues in lobe 1 of the WT and L651V LBD in complex with kainate. Unexpectedly, the comparison showed that the cleft is no more closed in the L651V mutant than in the WT domain (Fig. 3C). Compared with the corresponding glutamate-bound reference states, the WT:kainate cleft is rotated $6.7^{\circ}$, whereas the L651V:kainate cleft is rotated $6.8^{\circ}$. Although the difference may not be significant, the L651V:kainate complex is thus at least nominally more open than WT, despite being associated with higher relative efficacy.

The other standard LBD metric associated with activation is the separation between the Gly-Thr (GT) linkers that replace the two transmembrane domains in the recombinant LBD construct. This distance is thought to reflect the separation of the transmembrane helices in the intact receptor (Armstrong et al., 2003; Jin et al., 2003). Consistent with this model, comparison of the intermonomer Gly $\mathrm{C}_{\alpha}$ distances for the kainate- and glutamatebound complexes of the GluA4-L651V LBD reveals an increase from $34.9 \AA$ to $36.8 \AA$. However, the L651V:kainate separation is actually slightly smaller than the corresponding value obtained for the WT: kainate complex (35.6 $\AA$ ), in agreement with the cleft-closure observations.

\section{The L651V mutation results in interdomain twist}

Comparison of the WT and L651V LBD:kainate complexes from a face-on viewpoint reveals that the observed rms difference reflects a sideways motion or twist of lobe 2 of the L651V domain by $\sim 2.2^{\circ}$ (Fig. 3D). This motion is clearly not accounted for by a one-dimensional cleft-closure metric. We therefore considered an alternative two-component metric, based on principal-component analysis of agonist-induced interlobe motions (Bjerrum and Biggin, 2008). This involves measuring the separations between pairs of individual TM attachment points in the dimer, i.e., between Lys507 and Lys507' (M1 helices, GluA4 numbering) and Pro633 and Pro633' (M3 helices). As for the central Gly-Thr linker, both of these separations are actually slightly smaller for the GluA4-L651V:KA complex than for the GluA4-WT:KA complex (Table 2), despite the higher relative efficacy of kainate in the L651V channels.

Although the twist motion of lobe 2 present in the L651V:KA cocomplex originates from the Val residue, the largest movement is seen in the membrane proximal region of lobe 2 , near the linker to the transmembrane domains (TMDs). The lateral component of the displacement of the L651V LBD compared with the WT
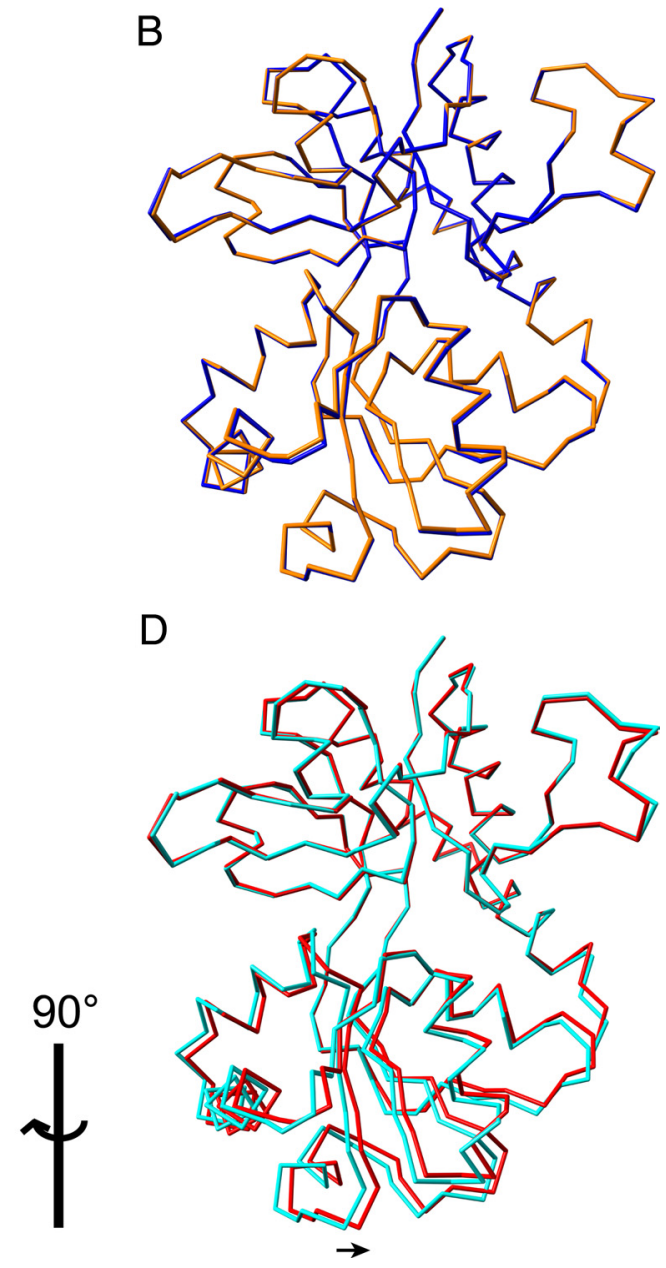

figure 3. The L651V mutation permits LBD interlobe twist, but no additional cleft closure. $A, B, C_{\alpha}$ traces are shown comparing the glutamate-bound conformations of the GluA4-WT (blue) and GluA4-L651V (gold) LBD structures. The cleft is seen in side (A) described in Materials and Methods.

Table 2. Interlobe distances between pairs of atoms in GluA4

\begin{tabular}{llll}
\hline GluA4 structures & Gly $_{\text {Linker }} C_{\alpha}$ & Lys507C $_{\alpha}$ & Pro633 $_{\alpha}$ \\
\hline GluA4-WT-LBD:Glu & 36.5 & 36.0 & 38.0 \\
GluA4-WT-LBD:KA & 35.6 & 34.6 & 35.6 \\
GluA4-L651V-LBD:Glu & 36.8 & 36.2 & 38.3 \\
GluA4-L651V-LBD:KA & 34.9 & 34.3 & 35.4 \\
\hline
\end{tabular}

LBD increases from $0.13 \AA$ at Gly654 to $0.55 \AA$ at residue Gly649, $0.65 \AA$ at the center of mass of lobe 2 , and $0.73 \AA$ at the most distal $\mathrm{C}_{\alpha}$ of the Gly within the GT linker. While this movement does not increase the interlobe distance between opposing pairs of TM attachment points in a dimer, it is still clearly associated with a significant increase in relative agonist efficacy (Fig. $2 B, C$ ).

An alternative representation of LBD conformational changes To visualize more precisely the conformational changes associated with agonist binding in the GluA4-WT and -L651V LBD structures, we reoriented the domain, viewing it along the first principal axis, corresponding approximately to the vertical axis in the standard edge-on or face-on perspectives (Fig. $4 A, B$ ). We then superimposed lobe 1 of the structure to be compared, and 


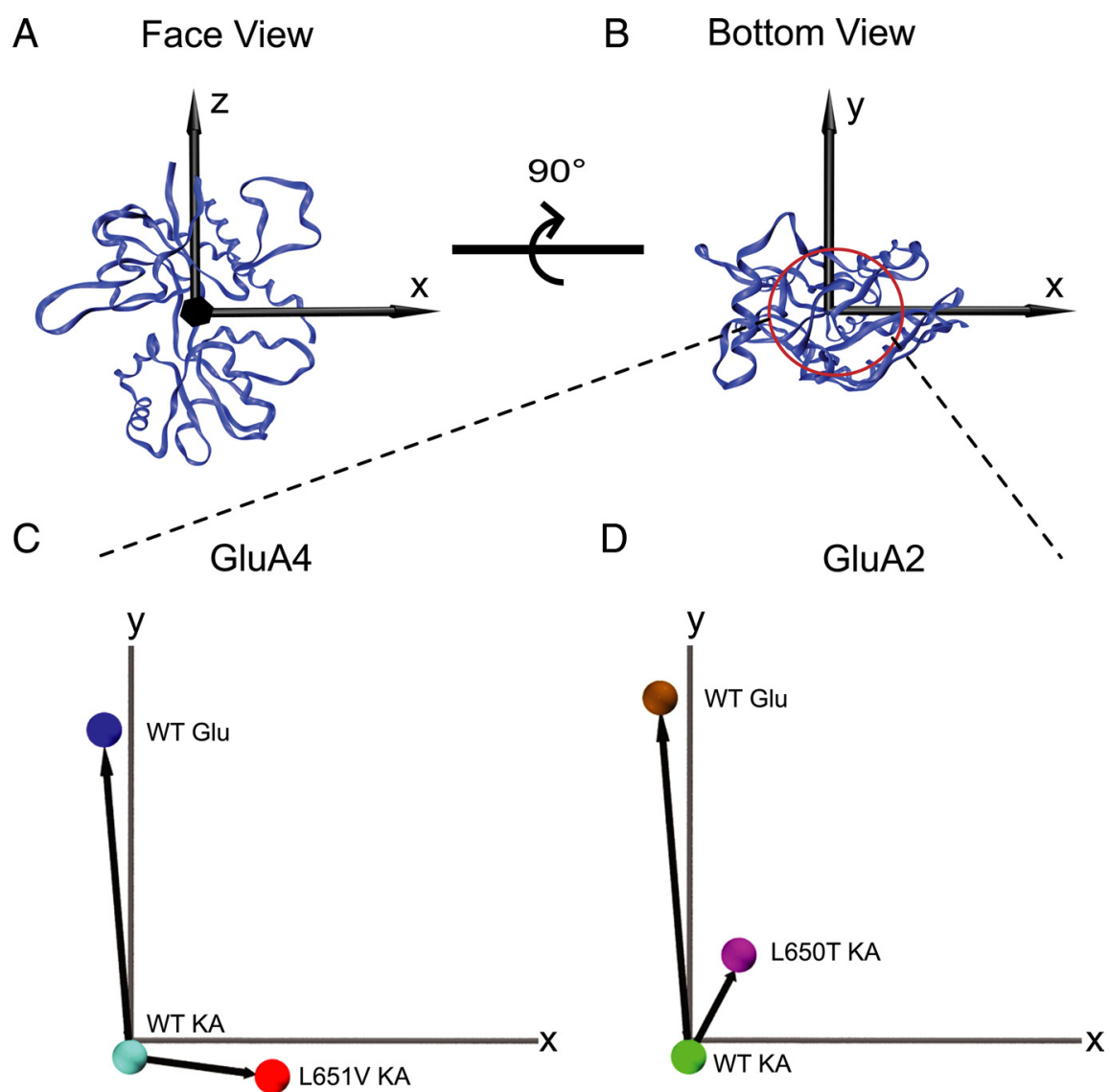

Figure 4. Principal-axis analysis reveals different conformational responses of GluA4-L651V and GluA2-L650T mutants. $\boldsymbol{A}, \boldsymbol{B}$, The structure of the kainate-bound GluA4-WT LBD was aligned to its principal axes as shown in face $(\boldsymbol{A})$ and bottom $(\boldsymbol{B})$ views of the domain. Changes along the first $(z)$ and second $(y)$ principal axes primarily reflect motions associated with cleft closure, whereas changes along the third principal axis $(x)$ reflect orthogonal motions. C, D, To visualize conformational changes associated with differences in relative efficacy, the center-of-mass displacements of lobe 2 of GluA4 (C) and GluA2 (D) LBD:agonist complexes are shown in projection along the bottom view corresponding to $B$, following superposition of lobe 1 . In each case the WT:KA complex is chosen as the origin. Note that displacements are not on the same scale as in $\boldsymbol{A}$ and $\boldsymbol{B}$. $\boldsymbol{C}$, The center of mass of lobe 2 of the GluA4-WT:Glu complex (dark blue) is displaced from the GluA4-WT:KA complex (light blue) primarily along the vertical second principal axis (y), whereas the GluA4-L651V:KA complex (red) is displaced along an orthogonal pathway close to the third principal axis $(x)$. D, The centers of mass of lobe 2 of the GluA2-WT:Glu complex (brown) and the GluA2-L650T:KA complex (purple) are both displaced from the GluA2-WT:KA complex (green) by a vertical shift primarily along the second cleft-closure axis (y).

plotted the vector connecting the centers of mass of lobe 2 in the two complexes. When this comparison was performed for the GluA4 WT LBD, the vector connecting the positions of lobe 2 in the kainate- and glutamate-bound WT structures points nearly vertically, along the second principal axis of the domain (Fig. 4C). This corresponds to the cleft-closure motion that brings the two lobes closer together. In contrast, the vector connecting the kainatebound positions of lobe 2 for the WT LBD and the L651V mutant is oriented at an oblique angle to the WT KA-Glu vector (Fig. 4C), reflecting the twisting motion seen in Figure $3 D$.

To compare our results with those obtained for the nearly isosteric GluA2 L650T mutant, we performed the same analysis on the published structures of the GluA2-WT and -L650T complexes with glutamate and kainate. As was seen for the GluA4 WT domain, the kainate-glutamate vector associated with the GluA2 WT domain is oriented vertically, along the cleft-closure axis (Fig. 4D). Consistent with the reported result of the L650T mutation (Armstrong et al., 2003) and in contrast with GluA4 (Fig. $4 C$ ), in this case the mutant vector lies nearly parallel to the WT vector (Fig. $4 D$ ).

\section{Stereochemical basis for L651V-induced twist}

To investigate how the L651V mutation permits a sideways displacement of lobe 2 relative to lobe 1 in the presence of kainate, we compared the stereochemical environment of the side chain in the WT and mutant complexes. Consistent with the branched, nonpolar character of both side-chain termini, in the mutant complex, the terminal valine isopropyl moiety occupies the hydrophobic pocket that contains the corresponding leucine moiety in the WT structure, with the $\mathrm{Val} \mathrm{C}_{\beta}$ nearly superimposed on the Leu $\mathrm{C}_{\gamma}$ position (Fig. 5A). In this sense, the mutant is behaving essentially as designed, although the terminal isopropyl moiety of the Val side chain also reorients around the $\mathrm{C}_{\beta}-\mathrm{C}_{\gamma}$ bond. However, since the WT Leu side chain is oriented parallel to the cleftclosure hinge axis, sliding the shorter $\mathrm{Val}$ into position results in a sideways movement of lobe 2 (Fig. $5 A$, arrow) rather than the upward movement seen in the L650T GluA2 structure.

The twisted conformation of the two lobes appears to be stabilized by a number of polar interactions. Hydrogen bonds are formed between the amide and carbonyl groups and two conserved waters and also between the main-chain carbonyl of $\mathrm{Val}$ and the side-chain hydroxyl group of the neighboring Thr residue. In addition, a number of surface-exposed salt bridges may be modestly strengthened by associated conformational rearrangements (D652:K657, E658:R661, E711:K764). Conversely, the twisted conformation is sterically inaccessible to the wild-type protein. If we model a Leu residue at position 651 in the L651V:kainate complex, we observe several clashes $(2.2-3.0 \AA)$ that are avoided by the shorter Val side chain (Fig. $5 B$ ).

The GluA2-L650T mutation is virtually isosteric with the GluA4-L651V mutation presented here. Like GluA4-L651V, the GluA2-L650T mutation increases the relative efficacy of kainate, yet previous analysis (Armstrong et al., 2003) revealed a $3^{\circ}$ increase in cleft closure with only a nominal amount of twist. The difference in conformational responses can clearly be seen in projections along the first principal axis (Fig. 4C,D). However, it is not caused by failure to accommodate the Thr side chain in the twisted structure. Modeling a Thr in place of the Val residue in the GluA4-L651V:kainate structure reveals no steric clash and a single potential hydrogen bond to the carbonyl oxygen of Thr684. Consistent with this, the Thr650 residue also does not reveal any steric clashes in the "extremely twisted" conformation observed for the GluA2-L650T LBD in complex with AMPA (PDB entry 1P1U, chain B) (Bjerrum and Biggin, 2008). Instead, it appears that the kainate-bound form of GluA2-L650T is stabilized in an untwisted state by an alternative hydrogen bond between the hydroxyl groups of Thr650 and Tyr702 (Phe703 in 
GluA4) (Fig. 5C), which may also account for the enhanced affinity of GluA2-L650T for kainate (Armstrong et al., 2003).

\section{Discussion}

Targeted engineering provided a key early test of the proposed roles of cleft closure and linker separation in regulating AMPAR agonist efficacy. Replacement of the binding-site Leu650 residue with a smaller Thr side chain in GluA2 relieved a steric clash with the kainate isopropenyl group and permitted a greater degree of cleft closure in the kainate complex compared with the WT domain. A corresponding enhancement of kainate relative efficacy was also observed (Armstrong et al., 2003).

However, several aspects of the GluA2L650T mutation were unusual. First of all, the mutation had unanticipated effects on the apparent affinities of a spectrum of agonists. Perhaps more importantly, it unexpectedly converted AMPA from a full to a partial agonist, even though crystal structures and later FRET (fluorescence resonance energy transfer) studies showed that AMPA still induced full cleft closure in the domain (Armstrong et al., 2003; Ramanoudjame et al., 2006). Subsequent analysis of the NR1 LBD (Inanobe et al., 2005) and of the GluA2-L686A mutation (Zhang et al., 2008) revealed other cases in which full cleft closure is associated with partial agonism, presumably caused by reduced selectivity for the closed versus open conformation of the cleft. In these cases, the proportion of receptors in the closed LBD conformation is reduced, but the increase in transmembrane domain separation associated with cleft closure is still assumed to provide the driving force for channel activation.

In addition to cleft closure, other LBD conformational changes have been identified as contributing to the overall extent of TMD separation. Even in early comparisons of GluA2:agonist structures, it was observed that the orientation of the rotation axis describing the cleft closure varied depending on the ligands involved (Armstrong and Gouaux, 2000; Hogner et al., 2002; Holm et al., 2005). Recently, an extensive principal-component analysis of published GluA2 LBD structures revealed three orthogonal motions, described as bending (i.e., closure), twist, and rock (Bjerrum and Biggin, 2008). Following closure, the twisting motion represented the second largest conformational change. Twist induced by partial agonists was associated with differential increases in the separation of helices M1 (smaller; associated with Lys506 in GluA2) and M3 (larger; associated with Pro632 in GluA2). Nevertheless, the structural transitions from antagonist to partial-agonist complexes and from partial- to full-agonist complexes were each associated with increases in both transmembrane separation metrics (Table 3). Bjerrum and Biggin (2008) further suggest that the twisting motion along the second eigenvector is associated with reduced efficacy. This assignment is based on the early proposal that the
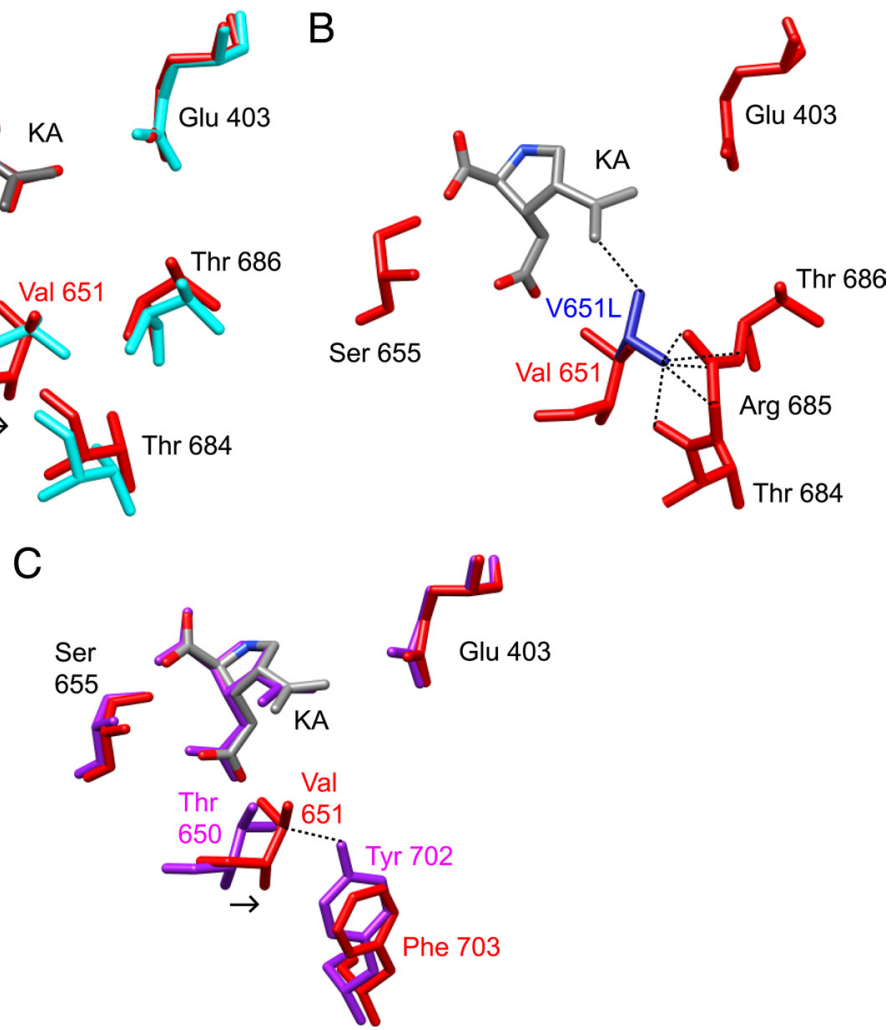

Figure 5. Stereochemical basis for L651V-induced LBD twist. $\boldsymbol{A}$, Following superposition of lobe 1 of the GluA4-WT:KA (cyan, gray) and GluA4-L651V:KA (red) complexes, side chains of the ligand-binding site are shown, together with the bound kainate 列

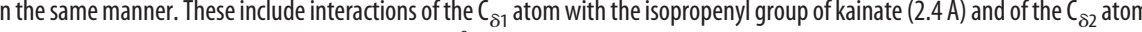
ith atoms of Thr684, Arg685, and Thr686 (2.2-3.0 A). C, Superposition of lobe 1 of the kainate-bound complexes of GluA2-L650T urple) and GluA4-L651V (GluA4 in red; kainate colored by atom type) shows the horizontal displacement of the GluA4-L651V complex compared with the isosteric GluA2-L650T mutant (arrow). The hydrogen bond between Thr650 and Tyr702 in the GluA2-L650T structure is shown as a dashed line.

Table 3. Interlobe distances between pairs of atoms in GluA2

\begin{tabular}{llll}
\hline GluA2 structures & Gly $_{\text {Linker }} C_{\alpha}$ & Lys506C $_{\alpha}$ & Pro632 $_{\alpha}$ \\
\hline 1FTJ (WT:Glu) & 36.9 & 36.3 & 38.6 \\
1FW0 (WT:KA) & 34.3 & 34.1 & 34.6 \\
1P1N (L650T:KA) & 35.3 & 34.6 & 36.0 \\
1P1U (L650T:AMPA AS form) & 35.6 & 35.0 & 37.0 \\
1P1Q (L650T:AMPA Zn form) & 36.9 & 36.3 & 38.7 \\
\hline
\end{tabular}

AS, Ammonium sulfate.

partially closed GluA2-L650T:AMPA LBD (PDB entry 1P1U, chain B) represents an inactive state (Armstrong et al., 2003).

Because of concerns about the unexpected effects of the GluA2-L650T mutation, and because the GluA4-WT domain already exhibited a greater degree of cleft closure than the GluA2-WT domain in complex with kainate (Gill et al., 2008), we set out to study a more stereochemically conservative mutation at the corresponding L651 site in GluA4. Consistent with previous binding studies of the GluA4-L651V LBD (Madden et al., 2004), we observed modest and roughly parallel shifts in the $\mathrm{EC}_{50}$ values for kainate and glutamate (Fig. 2A). As expected based on the elimination of a steric clash, the relative efficacy of kainate increased (Fig. 2B,C). However, in contrast to what was seen for the GluA2 LBD (Armstrong et al., 2003), we find no increase in cleft closure between the GluA4-WT and GluA4-L651V kainate complexes (Fig. 3C). 
The conformational change observed corresponds to an almost pure twist (Fig. 3D). It is of course possible that the isolated LBD does not precisely reflect the conformational changes that take place in the full-length receptor because of packing constraints imposed either in vivo by domain architecture or in vitro by the crystal lattice. A recent NMR study has revealed discrepancies between crystal and solution structures of GluA2:agonist complexes (Maltsev et al., 2008). However, our structure is consistent with spectroscopic data obtained from GluA4-LBD complexes in solution (Madden et al., 2004). In particular, the major difference between the vibrational spectra of the WT and L651V mutant LBDs in complex with kainate involved the thiol stretching vibration of Cys426. This mode exhibits a $13 \mathrm{~cm}^{-1}$ downshift in the GluA4-L651V:kainate complex compared with the WT: kainate complex, matching those observed in GluA4-WT complexes with AMPA and glutamate and consistent with a decrease in hydrogen-bonding distance. The crystal structure presented here indicates a hydrogen bond distance from the Cys426 sulfhydryl to the Ile477 carbonyl oxygen (3.93 $\AA$ ) that is shorter than that found in the WT:kainate complex $(4.06 \AA)$ and closer to the average value observed in the WT complexes with glutamate and AMPA (3.98 $\AA$ ). Although these shifts are at the limit of the resolution of our structure, they are consistent with the stereochemistry of the twisted binding site. As a result, it is reasonable to assume that the "sideways" rotation of lobe 2 reflects a genuine expansion of the conformational space available to the domain.

In previous analyses of twisted LBD structures, the predominant conformational change has been the concurrent increase in cleft closure, complicating assessment of the contribution of twist to agonist efficacy. In our case, the assignment of directionality is straightforward. The twist associated with the GluA4-L651V mutation in complex with kainate is parallel to that associated with the transition from the apo state to the partially closed GluA2-L650T:AMPA state. However, in contrast to the GluA2 interpretation (Bjerrum and Biggin, 2008), in GluA4 this twist is associated with increased relative efficacy. One explanation for the discrepancy is that the efficacy associated with the partially closed GluA2-L650T:AMPA structure may not be negligible, as originally proposed (Armstrong et al., 2003). Instead, the conversion of AMPA to a partial agonist may reflect reduced selectivity for the closed versus open cleft conformation(s) (Ramanoudjame et al., 2006; Zhang et al., 2008), even though the partially closed conformation retains significant activity.

Although it may therefore be tempting simply to assign reverse polarity to the twist:efficacy correlation, it seems more appropriate to question the model by which the LBD behaves as a linearly variable two-dimensional rheostat, converting closure and twist into specific TM separation metrics that trigger ever larger degrees of channel activation (Armstrong and Gouaux, 2000; Jin et al., 2003). In contrast to previous studies, the enhanced relative efficacy of kainate for the GluA4-L651V mutant occurs without an increase in any of the linker-separation metrics. Instead, the resulting conformational change can be described as a nearly pure rotational motion around the LBD dimer axis.

Considering that protein conformational changes are coarse grained, there may be very specific positions of the TM attachment points that trigger significant activation, and intermediate positions may be associated with reduced efficacy. To test this idea, we evaluated projections of lobe 2 center-of-mass motions for a variety of GluA2 LBD:agonist complexes (Fig. 6). Consistent with previous work, we find that the structures are distributed predominantly along an axis aligned closely with

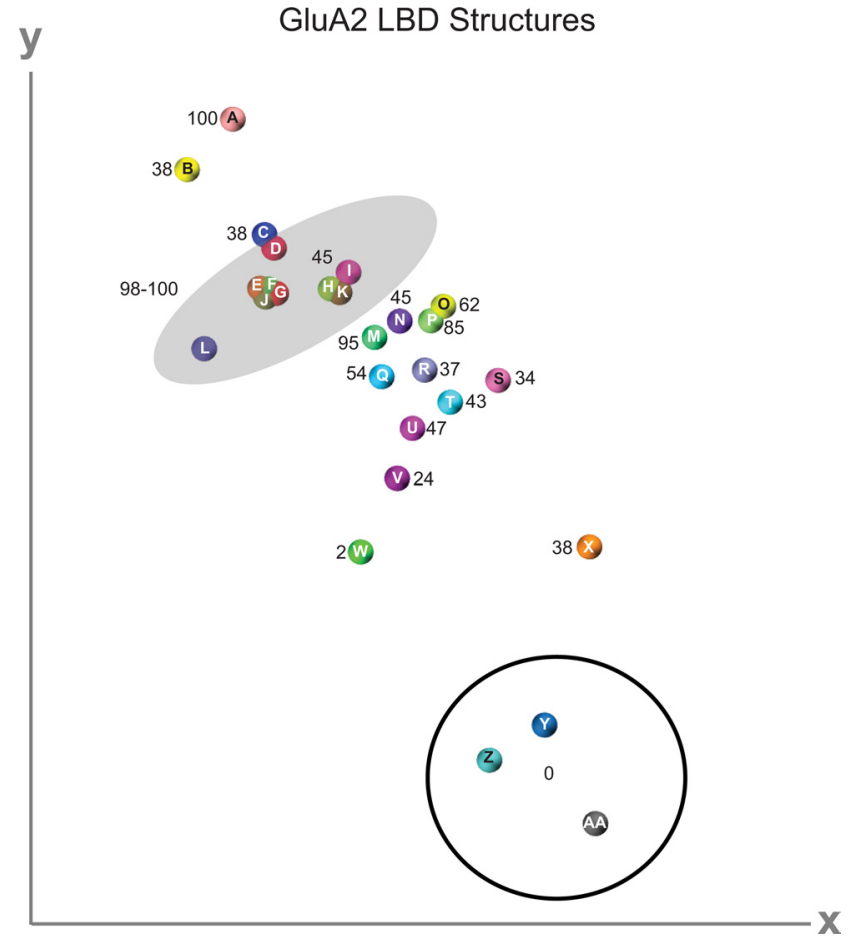

Figure 6. Nonlinear dependence of efficacy on cleft-closure and twist. As in Figure 4, the coordinates of lobe 1 of structures of GluA2 ligand complexes were individually aligned to those of the GluA2-WT:KA complex (1FW0) in principal-axis space. Spheres representing the center of mass of lobe 2 for each complex are shown in projection along the first principal axis with associated relative efficacy estimates (shown as percentage). Gray shading indicates a cluster of complexes showing near-maximal efficacy (98-100\%). The open circle shows the position of antagonist complexes. The center-of-mass displacements are oriented primarily along a single axis, but with significant orthogonal displacements. Efficacy does not vary smoothly or monotonically in either direction. The PDB entries used for the comparison (chain $A$, unless otherwise indicated) are indicated by single-letter codes: $A, 2 C M 0$ chain $B ; B, 1 P 1 U ; C, 1 P 1 W ; D, 1 P 10 ; E$, 1LBC; F, 1NNK; G, 1FTM; H, 1MM7;I, 1MQD; J, 1M5B; K, 1FTJ; L, 1M5E; M, 2AIX; N, 1SYI; 0, 1MQJ; P, 1M5C; $Q, 1 M Q I ; R, 1 M Q H ; S, 1 M Q G ; T, 1 S Y H ; U, 1 X H Y ; V, 1 P 1 N ; W, 1 F W 0 ; X, 1 P 1 U$ chain $B ; Y$, 1NOT; Z,1LB9; AA, 1 FTO.

cleft closure, but with a significant orthogonal component corresponding to twist or rock components. However, conformational changes associated with very different activities are surprisingly juxtaposed, both along and athwart the cleft-closure axis. Overall, it seems there may be "hot spots" of activation where the three-dimensional position of lobe 2 allows the transmembrane helices to assume a conformation that is compatible with ion flux. This discontinuous activation model is clearly distinct from a model in which efficacy is determined primarily as the linear combination of one or more orientational parameters.

Of course, attempts to interpret the contribution of specific conformational changes to receptor activation will ultimately require a knowledge of the nature of the changes occurring in the intact receptor. So far, analysis of AMPAR activation has focused on the LBD as a structurally accessible surrogate. Yet, although the LBD crystallizes in a physiologically relevant dimer, the crystallographically visualized pairs of TM attachment points still represent only one-half of the connections in a tetrameric receptor. The structure of an intact AMPAR, although currently available only in complex with a competitive antagonist (Sobolevsky et al., 2009), offers the perspective of comparing different agonist-bound structures in the future. Ultimately, the net force applied to the TM domains may well have a significant rotational or tangential component, in analogy to conformational changes 
associated with the opening of other pore-loop ion channels (Alam and Jiang, 2009; Bocquet et al., 2009; Hilf and Dutzler, 2009). Further complicating the physiological interpretation of such structures is the role of auxiliary subunits (Milstein and Nicoll, 2008). However, if it is true that relatively modest displacements of the TM attachment points can significantly affect agonist efficacy, then it is straightforward to envision how auxiliary subunits could mediate large-scale effects on both the nature and the magnitude of ligand-induced channel activity.

\section{References}

Alam A, Jiang Y (2009) High-resolution structure of the open NaK channel. Nat Struct Mol Biol 16:30-34.

Armstrong N, Gouaux E (2000) Mechanisms for activation and antagonism of an AMPA-sensitive glutamate receptor: crystal structures of the GluR2 ligand binding core. Neuron 28:165-181.

Armstrong N, Mayer M, Gouaux E (2003) Tuning activation of the AMPAsensitive GluR2 ion channel by genetic adjustment of agonist-induced conformational changes. Proc Natl Acad Sci U S A 100:5736-5741.

Bjerrum EJ, Biggin PC (2008) Rigid body essential X-ray crystallography: distinguishing the bend and twist of glutamate receptor ligand binding domains. Proteins 72:434-446.

Bocquet N, Nury H, Baaden M, Le Poupon C, Changeux JP, Delarue M, Corringer PJ (2009) X-ray structure of a pentameric ligand-gated ion channel in an apparently open conformation. Nature 457:111-114.

Braitenberg V, Schüz A (1998) Cortex: statistics and geometry of neuronal connectivity. Berlin: Springer.

Brünger AT, Adams PD, Clore GM, Delano WL, Gros P, Grosse-Kunstleve RW, Jiang JS, Kuszewski J, Nilges N, Pannu NS, Read RJ, Rice LM, Simonson T, Warren GL (1998) Crystallography and NMR system (CNS): a new software system for macromolecular structure determination. Acta Crystallogr D 54:905-921.

Collingridge GL, Olsen RW, Peters J, Spedding M (2009) A nomenclature for ligand-gated ion channels. Neuropharmacology 56:2-5.

Cowtan K (1994) dm: an automated procedure for phase improvement by density modification. Joint CCP4 and ESF-EACBM Newsletter on Protein Crystallography 31:34-38.

Derkach VA, Oh MC, Guire ES, Soderling TR (2007) Regulatory mechanisms of AMPA receptors in synaptic plasticity. Nat Rev Neurosci 8:101-113.

Dingledine R, Borges K, Bowie D, Traynelis SF (1999) The glutamate receptor ion channels. Pharmacol Rev 51:7-62.

Frydenvang K, Lash LL, Naur P, Postila PA, Pickering DS, Smith CM, Gajhede M, Sasaki M, Sakai R, Pentikainen OT, Swanson GT, Kastrup JS (2009) Full domain closure of the ligand-binding core of the ionotropic glutamate receptor iGluR5 induced by the high affinity agonist dysiherbaine and the functional antagonist 8,9-dideoxyneodysiherbaine. J Biol Chem 284:14219-14229.

Gill A, Madden DR (2008) Purification and crystallization of a non-GluR2 AMPA-receptor ligand-binding domain: a case of cryo-incompatibility addressed by room-temperature data collection. Acta Crystallogr F 64:831-835.

Gill A, Birdsey-Benson A, Jones BL, Henderson LP, Madden DR (2008) Correlating AMPA receptor activation and cleft closure across subunits: crystal structures of the GluR4 ligand-binding domain in complex with full and partial agonists. Biochemistry 47:13831-13841.

Hilf RJ, Dutzler R (2009) Structure of a potentially open state of a protonactivated pentameric ligand-gated ion channel. Nature 457:115-118.

Hogner A, Kastrup JS, Jin R, Liljefors T, Mayer ML, Egebjerg J, Larsen IK, Gouaux E (2002) Structural basis for AMPA receptor activation and ligand selectivity: crystal structures of five agonist complexes with the GluR2 ligand-binding core. J Mol Biol 322:93-109.

Holm MM, Naur P, Vestergaard B, Geballe MT, Gajhede M, Kastrup JS, Traynelis SF, Egebjerg J (2005) A binding site tyrosine shapes desensitization kinetics and agonist potency at GluR2: a mutagenic, kinetic, and crystallographic study. J Biol Chem 280:35469-35476.

Humphrey W, Dalke A, Schulten K (1996) VMD: visual molecular dynamics. J Mol Graph 14:33-38:27-38.

Inanobe A, Furukawa H, Gouaux E (2005) Mechanism of partial agonist action at the NR1 subunit of NMDA receptors. Neuron 47:71-84.

Jin R, Banke TG, Mayer ML, Traynelis SF, Gouaux E (2003) Structural basis for partial agonist action at ionotropic glutamate receptors. Nat Neurosci 6:803-810

Kabsch W (1976) A solution for the best rotation to relate two sets of vectors. Acta Crystallogr A 32:922-923.

Kabsch W (1993) Automatic processing of rotation diffraction data from crystals of initially unknown symmetry and cell constants. J Appl Crystallogr 26:795-800.

Madden DR (2002) The structure and function of glutamate receptor ion channels. Nat Rev Neurosci 3:91-101.

Madden DR, Cheng Q, Thiran S, Rajan S, Rigo F, Keinanen K, Reinelt S, Zimmermann H, Jayaraman V (2004) Stereochemistry of glutamate receptor agonist efficacy: engineering a dual-specificity AMPA/kainate receptor. Biochemistry 43:15838-15844.

Maltsev AS, Ahmed AH, Fenwick MK, Jane DE, Oswald RE (2008) Mechanism of partial agonism at the GluR2 AMPA receptor: measurements of lobe orientation in solution. Biochemistry 47:10600-10610.

Milstein AD, Nicoll RA (2008) Regulation of AMPA receptor gating and pharmacology by TARP auxiliary subunits. Trends Pharmacol Sci 29:333-339.

Pettersen EF, Goddard TD, Huang CC, Couch GS, Greenblatt DM, Meng EC, Ferrin TE (2004) UCSF Chimera-a visualization system for exploratory research and analysis. J Comput Chem 25:1605-1612.

Ramanoudjame G, Du M, Mankiewicz KA, Jayaraman V (2006) Allosteric mechanism in AMPA receptors: a FRET-based investigation of conformational changes. Proc Natl Acad Sci U S A 103:10473-10478.

Sobolevsky AI, Rosconi MP, Gouaux E (2009) X-ray structure, symmetry and mechanism of an AMPA-subtype glutamate receptor. Nature 462:745-756.

Zhang W, Cho Y, Lolis E, Howe JR (2008) Structural and single-channel results indicate that the rates of ligand binding domain closing and opening directly impact AMPA receptor gating. J Neurosci 28:932-943. 